\title{
ON SOME CUBIC MODULAR IDENTITIES
}

\author{
LI-CHIEN SHEN
}

(Communicated by Dennis A. Hejhal)

\begin{abstract}
Let $4 K$ and $2 i K^{\prime}$ be the periods of $\operatorname{sn} z$. By evaluating the functional equations $\operatorname{sn}^{2} z+\mathrm{cn}^{2} z=1$ and $k^{2} \mathrm{sn}^{2} z+\mathrm{dn}^{2} z=1$ at $z=i K^{\prime} / 3$, we deduce a set of cubic modular identities from which the familiar modular equation of degree 3 follows directly as a corollary.
\end{abstract}

The Jacobian elliptic functions (of modulus $k$ ) $\operatorname{sn}(z, k), \operatorname{cn}(z, k)$, and $\operatorname{dn}(z, k)$ are doubly periodic functions satisfying the fundamental identities

$$
\begin{aligned}
\operatorname{sn}^{2}(z, k)+\mathrm{cn}^{2}(z, k) & =1, \\
k^{2} \operatorname{sn}^{2}(z, k)+\operatorname{dn}^{2}(z, k) & =1 .
\end{aligned}
$$

It is customary to designate the periods of $\operatorname{sn}(z, k)$ as $4 K$ and $2 i K^{\prime}$, and the periods for $\operatorname{cn}(z, k)$ and $\operatorname{dn}(z, k)$ are respectively $4 K, 2\left(K+i K^{\prime}\right)$, and $2 K, 4 i K^{\prime}$. The values of those functions for certain $z$ are well known. For example, sn $K=1, \operatorname{cn} K=\operatorname{dn} K=0, \operatorname{sn}\left(i K^{\prime} / 2, k\right)=i / \sqrt{k}$, and $\operatorname{sn}(K / 2, k)=\left(1+k^{\prime}\right)^{-1 / 2}$. For other values see Chapter 22 of [4]. In this note, we will evaluate $\operatorname{sn}(z, k), \operatorname{cn}(z, k)$, and $\operatorname{dn}(z, k)$ at $z=i K^{\prime} / 3$ and link them, through the identities (1), to a set of cubic modular identities. We assume familiarity with the basic properties of theta functions, which can be found in Chapters 21 and 22 of [4].

Since our goal is to evaluate the elliptic functions at $z=i K^{\prime} / 3$, we therefore begin our discussion by recalling the definitions of the following elliptic functions:

$$
\begin{aligned}
& \operatorname{sn}(z, k)=\frac{\vartheta_{3}(\tau)}{\vartheta_{2}(\tau)} \frac{\vartheta_{1}\left(z \vartheta_{3}^{-2}(\tau) \mid \tau\right)}{\vartheta_{4}\left(z \vartheta_{3}^{-2}(\tau) \mid \tau\right)}, \\
& \operatorname{cn}(z, k)=\frac{\vartheta_{4}(\tau)}{\vartheta_{2}(\tau)} \frac{\vartheta_{2}\left(z \vartheta_{3}^{-2}(\tau) \mid \tau\right)}{\vartheta_{4}\left(z \vartheta_{3}^{-2}(\tau) \mid \tau\right)}, \\
& \operatorname{dn}(z, k)=\frac{\vartheta_{4}(\tau)}{\vartheta_{3}(\tau)} \frac{\vartheta_{3}\left(z \vartheta_{3}^{-2}(\tau) \mid \tau\right)}{\vartheta_{4}\left(z \vartheta_{3}^{-2}(\tau) \mid \tau\right)},
\end{aligned}
$$

where $\vartheta_{i}(\tau)=\vartheta_{i}(0 \mid \tau), i=2,3,4$.

Received by the editors January 28, 1992.

1991 Mathematics Subject Classification. Primary 33D10; Secondary 11B65.

Key words and phrases. Theta functions, quadratic transformation, modular identity. 
Using the facts that $K^{\prime}(\tau) / K(\tau)=-i \tau$ and $K(\tau)=\pi \vartheta_{3}^{2}(\tau) / 2$, we see that

$$
\begin{aligned}
& \operatorname{sn}\left(i \frac{K^{\prime}(\tau)}{3}, k\right)=\frac{\vartheta_{3}(\tau)}{\vartheta_{2}(\tau)} \frac{\vartheta_{1}(\pi \tau / 6 \mid \tau)}{\vartheta_{4}(\pi \tau / 6 \mid \tau)}, \\
& \operatorname{cn}\left(i \frac{K^{\prime}(\tau)}{3}, k\right)=\frac{\vartheta_{4}(\tau)}{\vartheta_{2}(\tau)} \frac{\vartheta_{2}(\pi \tau / 6 \mid \tau)}{\vartheta_{4}(\pi \tau / 6 \mid \tau)}, \\
& \operatorname{dn}\left(i \frac{K^{\prime}(\tau)}{3}, k\right)=\frac{\vartheta_{4}(\tau)}{\vartheta_{3}(\tau)} \frac{\vartheta_{3}(\pi \tau / 6 \mid \tau)}{\vartheta_{4}(\pi \tau / 6 \mid \tau)} .
\end{aligned}
$$

We now compute $\vartheta_{i}(\pi \tau / 6 \mid \tau)$ for $i=1,2,3,4$. For the sake of convenience, we will replace $\tau$ by $3 \tau$ and evaluate the quantities $\vartheta_{i}(\pi \tau / 2 \mid 3 \tau)$.

We begin by considering

$$
G(\tau)=\prod_{1}^{\infty}\left(1-q^{2 n}\right), \quad q=e^{\pi i \tau}, \operatorname{Im} \tau>0 .
$$

Recall that

$$
\vartheta_{4}(z \mid \tau)=\prod_{1}^{\infty}\left(1-q^{2 n}\right)\left(1-e^{2 i z} q^{2 n-1}\right)\left(1-e^{-2 i z} q^{2 n-1}\right) .
$$

Replacing $z$ by $\pi \tau / 2$ and $q$ by $q^{3}$, we obtain

(2) $\vartheta_{4}\left(\frac{\pi \tau}{2} \mid 3 \tau\right)=\prod_{1}^{\infty}\left(1-q^{6 n}\right)\left(1-q^{6 n-2}\right)\left(1-q^{6 n-4}\right)=\prod_{1}^{\infty}\left(1-q^{2 n}\right)=G(\tau)$.

To facilitate the computation further, we express $G(\tau)$ in terms of the elliptic moduli $k(\tau), k^{\prime}(\tau)$, and $K(\tau)$. We recall that

$$
K(\tau)=\frac{\pi}{2} \vartheta_{3}^{2}(\tau), \quad k(\tau)=\frac{\vartheta_{2}^{2}(\tau)}{\vartheta_{3}^{2}(\tau)}, \quad k^{\prime}(\tau)=\frac{\vartheta_{4}^{2}(\tau)}{\vartheta_{3}^{2}(\tau)} .
$$

Then

$$
G(\tau)=\left(\frac{2}{\pi^{3}} \frac{1}{\sqrt{q}} k^{\prime}(\tau) k(\tau) K^{3}(\tau)\right)^{1 / 6}, \quad q=e^{\pi i \tau} .
$$

The main advantage to expressing $G(\tau)$ in terms of these quantities is that we can apply the quadratic transformations:

$$
k(2 \tau)=\frac{1-k^{\prime}(\tau)}{1+k^{\prime}(\tau)}, \quad k^{\prime}(2 \tau)=\frac{2 \sqrt{k^{\prime}(\tau)}}{1+k^{\prime}(\tau)}, \quad K(2 \tau)=\left(\frac{1+k^{\prime}(\tau)}{2}\right) K(\tau) .
$$

Hence

$$
\begin{aligned}
G(2 \tau) & =\left(\frac{2}{\pi^{3}} \frac{1}{q} \frac{1-k^{\prime}(\tau)}{1+k^{\prime}(\tau)} \frac{2 \sqrt{k^{\prime}(\tau)}}{1+k^{\prime}(\tau)} \frac{\left(1+k^{\prime}(\tau)\right)^{3}}{8} K^{3}(\tau)\right)^{1 / 6} \\
& =\left(\frac{1}{2 \pi^{3}} \frac{1}{q} k^{2}(\tau) \sqrt{k^{\prime}(\tau)} K^{3}(\tau)\right)^{1 / 6}
\end{aligned}
$$

and

$$
\frac{G(2 \tau)}{G(\tau)}=\left(\frac{1}{4 \sqrt{q}} \frac{k(\tau)}{\sqrt{k^{\prime}(\tau)}}\right)^{1 / 6}
$$


We will evaluate $\vartheta_{i}(\pi \tau / 2 \mid 3 \tau)$ for $i=1,2,3$ and establish

\section{Proposition 1.}

$$
\begin{gathered}
\frac{\vartheta_{1}}{\vartheta_{4}}\left(\frac{\pi \tau}{2} \mid 3 \tau\right)=i\left(\frac{K(3 \tau)}{K(\tau)}\right)^{1 / 2}\left(\frac{k(3 \tau)}{k(\tau)}\right)^{1 / 4}, \\
\frac{\vartheta_{2}}{\vartheta_{4}}\left(\frac{\pi \tau}{2} \mid 3 \tau\right)=\left(\frac{K(3 \tau)}{K(\tau)}\right)^{1 / 2}\left(\frac{k(3 \tau) k^{\prime}(3 \tau)}{k(\tau) k^{\prime}(\tau)}\right)^{1 / 4}, \\
\frac{\vartheta_{3}}{\vartheta_{4}}\left(\frac{\pi \tau}{2} \mid 3 \tau\right)=\left(\frac{K(3 \tau)}{K(\tau)}\right)^{1 / 2}\left(\frac{k^{\prime}(3 \tau)}{k^{\prime}(\tau)}\right)^{1 / 4} .
\end{gathered}
$$

Prior to proving Proposition 1, we note several corollaries.

\section{Corollary 1.}

$$
\begin{aligned}
& \operatorname{sn}\left(i \frac{K^{\prime}(3 \tau)}{3}, k(3 \tau)\right)=\frac{i}{(k(\tau) k(3 \tau))^{1 / 4}}\left(\frac{K(3 \tau)}{K(\tau)}\right)^{1 / 2}, \\
& \operatorname{cn}\left(i \frac{K^{\prime}(3 \tau)}{3}, k(3 \tau)\right)=\left(\frac{k^{\prime}(3 \tau)}{k(3 \tau)}\right)^{1 / 2}\left(\frac{k(3 \tau) k^{\prime}(3 \tau)}{k(\tau) k^{\prime}(\tau)}\right)^{1 / 4}\left(\frac{K(3 \tau)}{K(\tau)}\right)^{1 / 2}, \\
& \operatorname{dn}\left(i \frac{K^{\prime}(3 \tau)}{3}, k(3 \tau)\right)=\frac{k(3 \tau)}{\left(k^{\prime}(\tau) k^{\prime}(3 \tau)\right)^{1 / 4}}\left(\frac{K(3 \tau)}{K(\tau)}\right)^{1 / 2} .
\end{aligned}
$$

\section{Corollary 2.}

$$
\begin{aligned}
& \text { (a) } \vartheta_{2}(\tau) \vartheta_{2}(3 \tau)=\frac{\vartheta_{4}^{3}(3 \tau)}{\vartheta_{4}(\tau)}-\frac{\vartheta_{3}^{3}(3 \tau)}{\vartheta_{3}(\tau)}, \\
& \text { (b) } \vartheta_{3}(\tau) \vartheta_{3}(3 \tau)=\frac{\vartheta_{4}^{3}(3 \tau)}{\vartheta_{4}(\tau)}-\frac{\vartheta_{2}^{3}(3 \tau)}{\vartheta_{2}(\tau)}, \\
& \text { (c) } \vartheta_{4}(\tau) \vartheta_{4}(3 \tau)=\frac{\vartheta_{3}^{3}(3 \tau)}{\vartheta_{3}(\tau)}-\frac{\vartheta_{2}^{3}(3 \tau)}{\vartheta_{2}(\tau)}
\end{aligned}
$$

Proof. From the identity $\operatorname{sn}^{2} v+\mathrm{cn}^{2} v=1$, we deduce that

$$
\left(\frac{K(3 \tau)}{K(\tau)}\right)\left[\frac{k^{\prime}(3 \tau)}{k(3 \tau)}\left(\frac{k(3 \tau) k^{\prime}(3 \tau)}{k(\tau) k^{\prime}(\tau)}\right)^{1 / 2}-\left(\frac{1}{k(\tau) k(3 \tau)}\right)^{1 / 2}\right]=1 .
$$

Expressing this identity in terms of theta functions, we obtain (a). Similarly, from the identity $k^{2} \operatorname{sn}^{2} v+\operatorname{dn}^{2} v=1$ we deduce (b). To prove (c), we replace $\tau$ by $\tau+1$ in (b) and recall that

$$
\begin{array}{ll}
\vartheta_{3}(\tau+1)=\vartheta_{4}(\tau), & \vartheta_{3}(3 \tau+3)=\vartheta_{4}(3 \tau), \\
\vartheta_{2}(\tau+1)=e^{\pi i / 4} \vartheta_{2}(\tau), & \vartheta_{2}(3 \tau+3)=e^{3 \pi i / 4} \vartheta_{2}(3 \tau) .
\end{array}
$$

Substituting this into $(b)$, the identity $(c)$ is established.

If we replace $\tau$ by $-1 / \tau$ and employ the imaginary transformation we obtain the following identities. 


\section{Corollary 3.}

$$
\begin{aligned}
& \vartheta_{2}(\tau) \vartheta_{2}(3 \tau)=\frac{1}{3}\left(\frac{\vartheta_{3}^{3}(\tau)}{\vartheta_{3}(3 \tau)}-\frac{\vartheta_{4}^{3}(\tau)}{\vartheta_{4}(3 \tau)}\right), \\
& \vartheta_{3}(\tau) \vartheta_{3}(3 \tau)=\frac{1}{3}\left(\frac{\vartheta_{2}^{3}(\tau)}{\vartheta_{2}(3 \tau)}-\frac{\vartheta_{4}^{3}(\tau)}{\vartheta_{4}(3 \tau)}\right), \\
& \vartheta_{4}(\tau) \vartheta_{4}(3 \tau)=\frac{1}{3}\left(\frac{\vartheta_{2}^{3}(\tau)}{\vartheta_{2}(3 \tau)}-\frac{\vartheta_{3}^{3}(\tau)}{\vartheta_{3}(3 \tau)}\right) .
\end{aligned}
$$

Clearly, from Corollary 2, we have

Corollary 4. $\vartheta_{4}(\tau) \vartheta_{4}(3 \tau)=\vartheta_{3}(\tau) \vartheta_{3}(3 \tau)-\vartheta_{2}(\tau) \vartheta_{2}(3 \tau)$.

Expressing the above corollary in terms of elliptic moduli, we obtain

Corollary 5 (Modular equation of degree 3 ).

$$
\sqrt{k(\tau) k(3 \tau)}+\sqrt{k^{\prime}(\tau) k^{\prime}(3 \tau)}=1 .
$$

It is interesting to note that the identity (b) in Corollary 2 is equivalent to

$$
\frac{K(3 \tau)}{K(\tau)}\left[\left(\frac{k^{\prime}(3 \tau)}{k^{\prime}(\tau)}\right)^{1 / 2} k^{\prime}(3 \tau)-\left(\frac{k(3 \tau)}{k(\tau)}\right)^{1 / 2} k(3 \tau)\right]=1
$$

Thus we can compute the quasi-period $K(3 \tau)$ from the given quasi-period $K(\tau)$. For example, it is well known that $k(i)=1 / \sqrt{2}=k^{\prime}(i)$ and $K(i)=$ $\Gamma^{2}(1 / 4) / 4 \sqrt{\pi}$. From (6) we easily obtain

(8) $k(3 i)=(\sqrt{2}-\sqrt[4]{3})\left(\frac{2-\sqrt{3}}{2}\right)^{1 / 2}$ and $k^{\prime}(3 i)=(\sqrt{2}+\sqrt[4]{3})\left(\frac{2-\sqrt{3}}{2}\right)^{1 / 2}$.

Substituting (8) into (7), we have

$$
K(3 i)=\frac{3}{4 \sqrt{2 \pi}}\left(3^{1 / 4}+3^{-1 / 4}\right) \Gamma^{2}\left(\frac{1}{4}\right) .
$$

In the notation of hypergeometric series, (9) is equivalent to

$$
\begin{aligned}
\vartheta_{3}^{2}(3 i) & ={ }_{2} F_{1}\left(\frac{1}{2}, \frac{1}{2} ; 1,(\sqrt{2}-\sqrt[4]{3})^{2}\left(\frac{2-\sqrt{3}}{2}\right)\right) \\
& =\sqrt{\frac{\pi}{2}} \frac{3}{8}\left(3^{1 / 4}+3^{-1 / 4}\right) \Gamma^{2}\left(\frac{1}{4}\right) .
\end{aligned}
$$

We now turn to the proof of Proposition 1. We recall that

$$
\vartheta_{3}(z \mid \tau)=\prod_{n=1}^{\infty}\left(1-q^{2 n}\right)\left(1+e^{2 i z} q^{2 n-1}\right)\left(1+e^{-2 i z} q^{2 n-1}\right)
$$


Replacing $\tau$ by $3 \tau$ and $z$ by $\pi \tau / 2$, we obtain

$$
\begin{aligned}
\vartheta_{3}(\pi \tau / 2 \mid 3 \tau) & =\prod_{n=1}^{\infty}\left(1-q^{6 n}\right)\left(1+q^{6 n-4}\right)\left(1+q^{6 n-2}\right) \\
& =\prod_{n=1}^{\infty} \frac{\left(1-q^{6 n}\right)^{2}\left(1-q^{12 n-4}\right)\left(1-q^{12 n-8}\right)\left(1-q^{12 n}\right)}{\left(1-q^{6 n}\right)\left(1-q^{12 n}\right)\left(1-q^{6 n-4}\right)\left(1-q^{6 n-2}\right)} \\
& =\frac{G(2 \tau)}{G(\tau)} \frac{G^{2}(3 \tau)}{G(6 \tau)} .
\end{aligned}
$$

Now using (5), we obtain

$$
\vartheta_{3}\left(\frac{\pi \tau}{2} \mid 3 \tau\right)=\left(\frac{q k(\tau)}{\sqrt{k^{\prime}(\tau)}} \frac{\sqrt{k^{\prime}(3 \tau)}}{k(3 \tau)}\right)^{1 / 6} G(3 \tau)
$$

and from (3) we have

$$
\frac{G(3 \tau)}{G(\tau)}=\left(\frac{1}{q} \frac{k(3 \tau)}{k(\tau)} \frac{k^{\prime}(3 \tau)}{k^{\prime}(\tau)} \frac{K^{3}(3 \tau)}{K^{3}(\tau)}\right)^{1 / 6}
$$

Thus

$$
\frac{\vartheta_{3}(\pi \tau / 2 \mid 3 \tau)}{\vartheta_{4}(\pi \tau / 2 \mid 3 \tau)}=\left(\frac{G(3 \tau)}{G(\tau)}\right)\left(\frac{G(3 \tau)}{G(6 \tau)}\right)\left(\frac{G(2 \tau)}{G(\tau)}\right)=\left(\frac{k^{\prime}(3 \tau)}{k^{\prime}(\tau)}\right)^{1 / 4} \sqrt{\frac{K(3 \tau)}{K(\tau)}} .
$$

Similarly, we have

$$
\begin{aligned}
\vartheta_{1}(\pi \tau / 2 \mid 3 \tau) & =i q^{1 / 4} \prod_{n=1}^{\infty}\left(1-q^{6 n}\right)\left(1-q^{6 n-1}\right)\left(1-q^{6 n-5}\right) \\
& =i q^{1 / 4} \prod_{n=1}^{\infty} \frac{\left(1-q^{2 n-1}\right)\left(1-q^{6 n}\right)}{\left(1-q^{6 n-3}\right)}
\end{aligned}
$$

We recall that

$$
\prod_{n=1}^{\infty}\left(1-q^{2 n-1}\right)=\left(2 q^{1 / 4} k^{\prime}(\tau) k^{-1 / 2}(\tau)\right)^{1 / 6}
$$

Thus

$$
\prod_{n=1}^{\infty} \frac{1-q^{2 n-1}}{1-q^{6 n-3}}=\left(\frac{1}{\sqrt{q}} \frac{k^{\prime}(\tau)}{k^{\prime}(3 \tau)} \sqrt{\frac{3 k(\tau)}{k(\tau)}}\right)^{1 / 6}
$$

Hence

$$
\begin{aligned}
\frac{\vartheta_{1}(\pi \tau / 2 \mid 3 \tau)}{\vartheta_{4}(\pi \tau / 2 \mid 3 \tau)} & =i q^{1 / 4} \frac{G(3 \tau)}{G(\tau)}\left(\frac{1}{\sqrt{q}} \frac{k^{\prime}(\tau)}{k^{\prime}(3 \tau)} \sqrt{\frac{k(3 \tau)}{k(\tau)}}\right)^{1 / 6} \\
& =i\left(\frac{k(3 \tau)}{k(\tau)}\right)^{1 / 4}\left(\frac{K(3 \tau)}{K(\tau)}\right)^{1 / 2} .
\end{aligned}
$$

Clearly $\left(\vartheta_{2} / \vartheta_{4}\right)(\pi \tau / 2 \mid 3 \tau)$ can be treated in a similar fashion; we therefore omit its proof.

It should be pointed out that there exist many different proofs of the modular equation of degree 3 (i.e., Corollary 5). In [2, pp. 214-223] Hardy gives three 
different proofs of this identity. The first proof due to Legendre is based on the transformation of elliptic integrals of the first kind, the second proof depends on direct manipulation of theta series, and the third proof due to Ramanujan involves the Lambert series for $\vartheta_{i}(\tau) \vartheta_{i}(3 \tau), i=2$, 4. A proof, which uses the modular machine, can be found in Rankin's book on modular forms [3, p. 232]. Our proof follows directly from the identities in Corollary 2, which are of some interest in themselves. In particular, in view of (7), they can be regarded as analogues of the familiar quadratic transformation [4, p. 507]

$$
\frac{K(2 \tau)}{K(\tau)} \cdot \frac{2 k^{1 / 2}(2 \tau)}{k(\tau)}=1
$$

Finally, we add that recently Borwein, Borwein, and Garvan [1] also derived a set of cubic modular identities and studied the connection of $\vartheta_{4}^{3}(3 \tau) / \vartheta_{4}(\tau)$ and $\vartheta_{4}^{3}(\tau) / \vartheta_{4}(3 \tau)$ with the hypergeometric series ${ }_{2} F_{1}\left(\frac{1}{3}, \frac{2}{3} ; 1 ; z\right)$.

\section{REFERENCES}

1. J. M. Borwein, P. B. Borwein, and F. G. Garvan, Some cubic modular identities of Ramanujan, Trans. Amer. Math. Soc. (to appear).

2. G. H. Hardy, Ramanujan, Chelsea, New York, 1978.

3. R. Rankin, Modular forms and functions, Cambridge Univ. Press, London and New York, 1977.

4. E. T. Whittaker and G. N. Watson, A course of modern analysis, 4th ed., Cambridge Univ. Press, London and New York, 1958.

Department of Mathematics, University of Florida, Gainesville, Florida 32611-2082

E-mail address: shen@math.ufl.edu 